\title{
Digital Sunflower: the potential of eco-oriented responsivity in the design process
}

XXIV International Conference of the Iberoamerican Society of Digital Graphics Medellin | Colombia

\author{
Bruno Massara Rocha \\ Universidade Federal do Espírito Santo | Brasil | bmassara@gmail.com \\ Raquel Souza Celestino \\ Universidade Federal do Espírito Santo | Brasil | raquelcelestino05@gmail.com \\ Kiany Ferreira Damascena Silva \\ MULTIVIX | Brasil | kianyfd@hotmail.com \\ Isabella Soares Galimberti \\ Universidade Federal do Espírito Santo | Brasil | isabella.galimberti@gmail.com
}

\begin{abstract}
In the face of the global energy crisis, this work presents the results of an effort to build an alternative device to improve the production of clean and renewable energy with high technology and low cost. The project titled Artificial Sunflower is a Arduino based opensource solar tracker conceived to enhance the performance of photovoltaic modules. It was used a research by design methodology to develop several prototypes using $3 \mathrm{~d}$ printing and laser cutting. The results include hardware and software information used to build and configurate the system.
\end{abstract}

Keywords: Solar tracker; Opensource; Arduino; Cybernetics; Sustainability;

\section{INTRODUÇÃO}

Diante da crise energética mundial (Bondarik et al., 2018), este trabalho apresenta os resultados de um esforço em construir uma alternativa técnica para aprimorar a produção de energia limpa e renovável com soluções que combinam alta tecnologia e baixo custo. Em um país com enorme potencial de produção de energia solar em função de sua localização e extensão geográfica, não há razões para que não haja investimentos no desenvolvimento tecnológico nacional orientado neste sentido, em especial no campo da arquitetura, engenharia e design industrial. Acreditamos ser possível não apenas desenvolver novos mecanismos de geração de energia limpa, mas também aprimorar aqueles existentes por meio da cibernética, da robótica e dos processos de fabricação digital. O trabalho aqui apresentado é resultado de um processo de concepção, programação, fabricação e testes de um seguidor solar responsivo ativo (Oliveira, 2008) categoria 3 (Blaszczak, 2017), denominado Girassol Digital, cujo objetivo é ampliar a performance de módulos fotovoltaicos e assim aumentar o rendimento na geração de energia solar, trazendo contribuições para projetos de arquitetura, design com aplicações em edifícios e equipamentos públicos.

Conceitualmente, o Girassol Digital é fruto de um projeto de inspiração biomimética (Benyus, 2003) relacionado ao princípio heliotrópico presente em muitas espécies vegetais como o Heliantus annuus. O princípio heliotrópico corresponde à capacidade do organismo vegetal inclinar sua estrutura física para melhor captar os raios solares e maximizar a fotossíntese. No projeto aqui apresentado a simulação do princípio heliotrópico fo realizada por meio de um mecanismo cibernético de primeira ordem (Pangaro \& Dubberly, 2011) cuja base é um microcontrolador Arduino UNO. No artigo estarão presentes informações detalhadas sobre os componentes utilizados, a representação gráfica do mecanismo, especificações técnicas, programação utilizada, imagens e recursos operacionais especialmente desenvolvidos para concretizá-lo tecnicamente. Os principais resultados alcançados são: uma programação computacional estável, leve e adaptada para versões tradicionais da plataforma Arduino; um mecanismo cibernético funcional e autônomo, capaz de se auto regular em qualquer localização geográfica e, assim sendo, independente de reprogramações futuras; um sistema produzido a baixo custo, totalmente opensource e passível de compartilhamento e replicação.

A influência da natureza e dos sistemas vivos em projetos de arquitetura é ancestral conforme revela pesquisas realizadas por Porthoghesi (1993) e Rebello (2000) e tocam inúmeros aspectos relacionados à adaptabilidade, economia de recursos, performance estrutural, além de fatores estéticos essenciais para a sobrevivência de muitas espécies como fator de defesa, reprodução e alimentação. Para além das inúmeras possíveis relações descritas pelos autores, o projeto do Girassol Digital articula-se não apenas com os estudos da biomimética abordados em Benyus (2003) mas também com o campo da biônica, ciência dedicada à investigação de sistemas que apresentam características específicas de sistemas 
naturais (ARRUDA, 2018) como é o caso do princípio heliotrópico adotado.

\section{OBJETIVOS E FUNDAMENTAÇÃO TEÓRICA}

O objetivo deste trabalho é demonstrar como construir, programar e simular o princípio heliotrópico e incorporá-lo a sistemas de captação de energia fotovoltaica. Ao contrário dos seguidores solares programados (Ramos et al., 2020) este trabalho desenvolve um sistema autorregulador capaz de identificar automaticamente a fonte de luz solar e posicionar os módulos fotovoltaicos sempre na posição ideal para o melhor aproveitamento. Com esta função, é possível construir um sistema que pode ser instalado em qualquer localização geográfica sem necessidade de reprogramação. Este é, sem dúvida, uma contribuição essencial para o projeto de sistemas responsivos porque dá autonomia para o equipamento perceber e se adaptar ao ambiente autonomamente.

Para ativar o sistema responsivo teve-se como objetivo desenvolver uma investigação na síntese de códigos de programação opensource que pudessem ser aprimorados e adaptados às demandas de controle do sistema. A programação é fator essencial para se criar a singularidade do sistema em função de suas características, especificações e objetivos de comportamento. Esta etapa da pesquisa foi viabilizada pela grande disponibilidade de programações análogas em sites e fóruns de discussão sobre computação física, linguagens de programação e compartilhamento de dados digitais no universo tanto dos softwares quanto hardwares.

Outro elemento direcionador presente nos objetivos do projeto foi conceber, modelar e prototipar não apenas o corpo do mecanismo (que inclui peças, estruturas e conectores), mas também os equipamentos necessários para testar seu funcionamento (heliodom). O processo de prototipagem considerou o uso coerente dos recursos materiais que incluiu o desperdício próximo a zero, uso de materiais biodegradáveis como o PLA e 0 reaproveitamento de recursos nos diversos ciclos de aperfeiçoamento do protótipo.

\section{FUNDAMENTAÇÃO TEÓRICA}

O sistema responsivo artificial apresentado neste trabalho possui tem uma inspiração nos sistemas naturais que é a sua capacidade de adaptação ao meio ambiente. Os fundamentos teóricos que permitem conceber sistemas artificiais com comportamento semelhante aos organismos vivos podem ser encontrados na cibernética, em especial nos princípios do feedback. De acordo com Wiener (1970) a cibernética é a ciência da comunicação e do controle, que abarca tanto organismos quanto máquinas, e que tem no feedback sua instância de agenciamento operativo. Por meio de ciclos recursivos de programação que incluem a percepção do ambiente por meio de sensores, a conversão de dados analógicos em digitais, o cálculo algorítmico e o controle da posição dos próprios sensores, o sistema consegue simular o comportamento de um organismo que segue uma fonte de luz. A recursividade é um conceito chave para o desenvolvimento de sistemas responsivos porque explica teoricamente o comportamento de um sistema artificial digital. Ela é a condição cíclica (Rocha, 2015) que uma programação digital possui de estar permanentemente avaliando seu estágio atual em comparação com as novas entradas de informação. À medida que novas informações chegam ao sistema por meio dos sensores, um conjunto de regras de cálculo são aplicadas, em sequência, para verificar, de forma lógica, a melhor regulagem para as variáveis de controle. Um dos desafios maiores da programação encontra-se na redação lógica destas regras para fazer com que o sistema "entenda" o que ele está "percebendo" e que tipo de ação deve tomar para que atinja seu objetivo programado. Neste sentido, a condição de permanente alteração do ambiente externo, como é o caso da posição solar, precisa ser transferida para o sistema artificial para que ele entre em concordância com o meio que optou por interagir. 0 Girassol digital pode ser considerado um mecanismo cibernético autorregulador de primeira ordem (Pangaro \& Dubberly, 2010) uma vez que sua programação opera a partir da detecção e correção de erros. Este processo também pode ser definido como convergência.

Conceitos relacionados à teoria cibernética são essenciais para o desenvolvimento de um projeto de sistema artificial de inspiração biomimética. Cabe explicar que tocamos aqui em um ponto específico da grande área de abrangência que é a biomimética. Soares \& Arruda (2018) explicam que os estudos de base biomimética pode incorporar diversos tipos de analogias com a natureza: funcional, morfológica, simbólica, com inúmeras possibilidades de desdobramentos. No caso aqui apresentado encontra-se em evidência uma busca por uma analogia funcional motora de um girassol representada pelo princípio heliotrópico. Não se trata de uma analogia morfológica, nem simbólica e nem de uma funcionalidade avançada como a realização de processos de fotossíntese. A analogia funcional tenta compreender qualidades específicas de operação de um sistema natural para transpô-lo em artefatos artificiais. Dada a complexidade inerente aos sistemas vivos, as relações de analogia biomiméticas poderiam se basear em princípios químicos, orgânicos, estruturais, reprodutivos, evolutivos e muitas outras formas de comportamento e ação presentes nos seres vivos. Obviamente, por questões de ordem metodológica e técnica, o recorte aqui delimitado recorre ao heliotropismo em sua instância mais básica que é o agenciamento do corpo do sistema em função da sua percepção, ou melhor sensoriamento, da fonte luminosa.

O desenvolvimento de sistemas artificiais que adotam recursos computacionais para simular comportamento pertence a um campo multidisciplinar denominado computação física (O'sullivan \& Igoe, 2004). Entende-se por computação física a construção de interfaces com capacidade associativa entre dados físicos (analógicos) e informações (digitais) através de um processo denominado transdução. Segundo O'Sullivan \& Igoe (2004), o processo de transdução consiste na conversão de formas de energia física em tensão elétrica variável, e recebe este nome devido ao elemento de conversão chamado transdutor. O principal componente eletrônico do processo de transdução é o microcontrolador, um dispositivo eletrônico que converte sinais analógicos em digitais e vice-versa. Todas as relações de conversão entre sinais analógicos e digitais são coordenadas por 
uma programação específica que fica armazenada no microcontrolador acoplado ao equipamento.

A programação é responsável pelo comportamento heliotrópico do mecanismo cibernético. Ela está baseada em uma sequência de cálculos em loop, ou seja, o sistema está permanentemente reavaliando sua condição interna para responder à demanda externa. Nos termos de Noble (2009) os loops da programação são responsáveis por manter relações de feedback do sistema com ele mesmo. Sem os loops um sistema não consegue se autorregular porque ele não saberia exatamente o que está fazendo. A programação de interfaces responsivas instaura um novo nível de complexidade ao projeto que é sua capacidade de ser variável em função das contingências contextuais (omitido, xxxx). Na verdade, o conceito de programa não é tão distante da prática projetual, uma vez que ele pode fazer referência a uma sequência de funções a serem atendidas. Mas com a programação digital ele assume uma condição ampliada fazendo referência a um sistema de relações e interdependências entre um conjunto de variáveis.

Em termo conceituais, podemos afirmar que o Girassol digital é, portanto, um sistema cibernético responsivo autorregulador, derivado de um projeto de computação física, que simula o comportamento de um girassol através de uma analogia funcional biomimética.

\section{METODOLOGIA}

A metodologia utilizada foi inspirada na Design Science Research caracterizada por sua natureza prescritiva e projetual com o objetivo de desenvolver soluções práticas ou aprimorar soluções para artefatos e sistemas existentes (Dresch et al., 2015). O propósito dessa metodologia é produzir sistemas que ainda não existem, ou que não estão disponíveis ou acessíveis para a realidade e o contexto em que será instalado. Neste sentido, tem objetivo prescritivo e utiliza como suporte fundamental o desenvolvimento projetual. Por meio do projeto, espera-se com esta metodologia desenvolver artefatos que permitam soluções satisfatórias para problemas práticos da realidade externa. Os resultados são avaliados qualitativamente e quantitativamente e busca criar aplicações e experientos que permitam não apenas compreender a realidade mas transformá-la. A aplicação da metodologia inclui três etapas: estruturação do projeto, construção do protótipo e avaliação por meio de ciclos de aprimoramento.

\section{ETAPA DE ESTRUTURAÇÃO}

A primeira etapa da metodologia é denominada estruturação e consistiu na delimitação do problema e de possíveis caminhos de aproximação. Foram realizados levantamentos de projetos análogos e o mapeamento das soluções técnicas opensource de baixo custo. A estrutura física foi baseada em um modelo composto por quatro sensores fotossensíveis LDR, dispostos em uma estrutura em formato de cruz extrudada, com dois eixos de giro operados por dois servo-motores, todos controlados por uma placa Arduino UNO. O modelo em formato de cruz foi recorrente em pesquisa de referências realizada em sites como Instructables, Youtube e em pesquisas no Google com o dado de busca "Arduino solar tracker" e "seguidor solar Arduino". A opção pela plataforma Arduino se deu em função da sua disponibilidade no laboratório de projetos anteriores.

A programação utilizada foi adaptada de Atomo (2020) e consiste em um script em Java para plataforma Arduino. Esta programação funciona da seguinte maneira: os valores de iluminação captados pelos quatro sensores são comparados entre si tendo como objetivo a manutenção dos valores sempre iguais. Caso a posição da fonte de luz provoque sombra em qualquer um dos sensores, os motores são acionados para corrigir a posição do equipamento de modo que o sensor volte a ser diretamente iluminado. O objetivo do código de programação é controlar o corpo do mecanismo para que ele esteja sempre em posição perpendicular ao sol e, consequentemente, com iluminação plena em todos os sensores simultaneamente. A estrutura de ligação dos sensores, resistores, motores e fontes de energia pode ser ilustrada pelo diagrama a seguir:

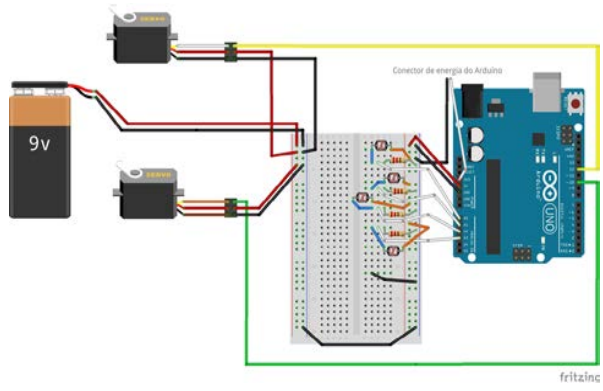

Figura 01: diagrama de distribuição das peças e ligações dos componentes eletrônicos utilizados. Fonte: autores

\section{ETAPA DE CONSTRUÇÃO}

A segunda etapa da metodologia é denominada construção ou transformação e consiste na aplicação das soluções levantadas que se mostram potencialmente mais viáveis em um protótipo inicial. Esta etapa tem como objetivo iniciar os primeiros testes práticos de construção, configuração e solução de problemas práticos em ambiente laboratorial.

Para a concepção do corpo do mecanismo foram adotados softwares de modelagem 3D como Sketchup e Rhinoceros. O corpo é composto por três partes principais que são: a haste principal, os conectores e o suporte para as placas (Figura 02). Todos os componentes do equipamento foram exclusivamente modelados pelos pesquisadores do Grupo de Pesquisa Conexão VIX para atender as demandas do projeto e foram também fabricados digitalmente utilizando os recursos de impressão 3D e corte a laser. Como componentes adicionais estão presentes uma bateria estacionária recarregável de $9 \mathrm{v}$, uma protoboard de 400 pontos e placas de suporte para os módulos fotovoltaicos. As placas de suporte possuem pontos de fixação da haste principal e foram dimensionadas para atender um cálculo inicial de 04 mini painéis fotovoltaicos de silício monocristalino de $11,5 \times 8,5 \mathrm{~cm}(9 \mathrm{v})$. A composição final do equipamento combinou toda a parte elétrica-eletrônica com peças impressas em $3 d$ resultando em um mecanismo vertical conforme ilustra a Figura 03 


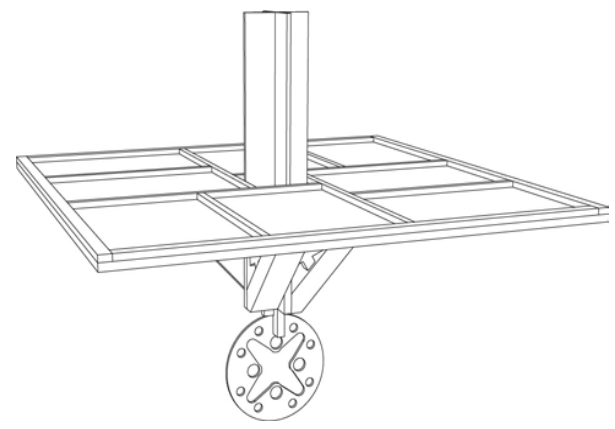

Figura 02: visão tridimensional dos componentes projetados do sistema Girassol Digital. Fonte: autores
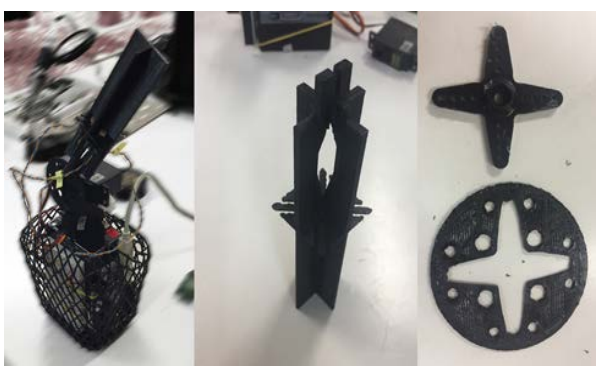

Figura 03: Impressões 3d dos componentes projetados: corpo em diagrid, haste para posicionamento dos sensores e encaixe para os motores. Fonte: autores

É possível visualizar que a parte inferior do equipamento concentra a bateria, a placa Arduino e a protoboard, e ela é envolvida por uma membrana impressa em $3 d$ no formato de diagrid (cor preta) que tem a função de proteger as ligações elétricas e permitir o manuseio do equipamento. Esta pele foi concebida no software de modelagem paramétrica Rhinoceros tomando como referência as medidas específicas do conjunto dos sistemas eletrônicos do Girassol Digital. O código de programação apresenta as seguintes variáveis: a) a intensidade de luz captada em cada um dos quatro sensores fotossensíveis (valores mapeados de 0 a 1000); b) o ângulo de rotação para os dois servo-motores (valores de 0 a 180 graus); c) o intervalo de tempo para o loop de leitura e comparação dos valores (100 a 1000 milissegundos); d) a média comparativa entre a intensidade de cada sensor fotossensível; e) a diferença entre as médias dos sensores. 0 objetivo da programação é monitorar a intensidade de luz de cada um dos sensores e, em caso de alteração, identificar por meio da comparação das médias, em que posição ela ocorreu. Esse procedimento visa reconhecer em que quadrante da haste do equipamento ocorreu o sombreamento do sensor. Identificado este quadrante, a programação altera o ângulo de rotação dos motores para que ele possa levar a haste em direção oposta e retomar a iluminação direta do sensor que se encontra no quadrante sombreado. Dessa forma, a cada intervalo de tempo pré-definido (loop), o sistema avalia sua condição interna (recursividade) e compara com as condições externas propondo ou não ajustes em sua condição interna de forma autorreguladora.

\section{ETAPA DE AVALIAÇÃO E APRIMORAMENTO}

A terceira e última etapa da metodologia é denominada avaliação e aprimoramento e consiste na ativação do mecanismo para testes de funcionamento e a construção de estruturas auxiliares para dar suporte, como é o caso do heliodom. Os dados obtidos nesta etapa serão utilizados para avaliar a performance do sistema responsivo, realizar sua documentação e disponibilizar os dados em uma plataforma online. Os principais critérios utilizados para avaliar a performance foram: a) tempo de resposta para o posicionamento do sistema em relação à fonte de luz; b) estabilidade da estrutura durante movimentação; c) resistência das ligações durante a movimentação; d) resistência dos componentes durante a movimentação. Para a realização de testes foi construído um protótipo de heliodom simplificado que auxilia a simulação da trajetória solar (Fig.04).

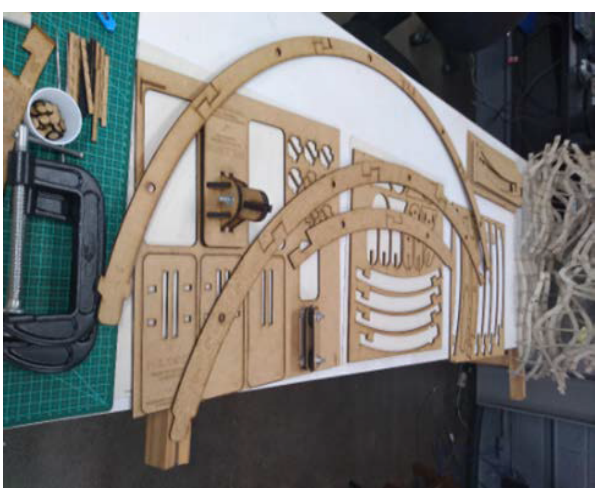

Figura 04: Componentes projetados e cortados a laser antes da montagem. Fonte: autores

O modelo construído simula a trajetória solar no período do equinócio. O projeto do heliodom foi realizado no Sketchup e no AutoCAD e construído com placas de MDF $3 \mathrm{~mm}$ cortado a laser. Os encaixes dos arcos de madeira foram inspirados no projeto Wikihouse. A Wikihouse é um sistema construtivo de código-aberto desenvolvido para o uso em madeira e totalmente baseado em encaixes e travamentos. Os encaixes em formato de "s" foram adotados para unir as peças que compõem o arco principal do heliodom. Os demais encaixes foram resolvidos com padrão macho-fêmea e auxílio de barras rosqueadas.

\section{ANÁLISE DOS RESULTADOS}

Não há número significativo de aplicações do princípio heliotrópico em projetos de arquitetura. Os exemplos identificados ao longo dos levantamentos de dados demonstram que seguidore solares são comumente passivos e programados para simular e sincronizar sua orientação com a trajetória solar. Estes modelos não são responsivos e não utilizam nenhum recurso cibernético para funcionarem. As análises aqui descritas visam avaliar a performance funcional e construtiva do equipamento. Em termos construtivos foram analisados: materiais e técnicas de produção, em vista da qualidade dos encaixes, movimentação, acabamento, tempo de produção, equilíbrio geral do sistema. Em termos 
funcionais, os principais pontos avaliados são: tempo de resposta e estabilidade da programação.

Com relação às estratégias de construção, o processo de modelagem e impressão 3d viabilizou a concepção detalhada de componentes e sua rápida prototipagem com qualidade elevada. Ao contrário dos modelos identificados na primeira fase do projeto, desenvolvidos com peças de papelão, isopor ou lego, o Girassol Digital obteve um nível de qualidade superior, com maior precisão, com melhor operatividade para o desenvolvimento de novas versões, considerando que nem sempre o design inicial de uma peça atenda à demanda esperada. A prototipagem digital permitiu um ganho de complexidade no desenho dos componentes, como encaixes entre os motores, encaixe do motor com a haste, encaixes entre sensores e haste, e o comprimento final da haste para gerar uma diferenciação de sombra adequada. Por trás do desenho final dos componentes há um histórico de várias versões, com diferentes ajustes e adaptações, até se obter uma peça leve, de fácil impressão, resistente e adequada ao tipo de ligação e função no sistema. Estas versões anteriores foram sendo catalogadas e arquivadas durante todo o processo. Para a versão atual do sistema todos os componentes impressos em 3d utilizaram única e exclusivamente a quantidade necessária de material, e atenderam à demanda pelo desperdício zero. O processo de concepção das peças ocorrer com a separação dos componentes: conector de motor, base da haste, topo da haste e barras de suporte dos módulos fotovoltaicos. Apesar de terem sido concebidas juntas em um mesmo arquivo digital, as peças foram impressas separadamente e depois montadas e coladas.

Para a construção do heliodom foi utilizado o processo de corte a laser. Ao contrário da impressão $3 d$, o corte é um processo subtrativo que parte de uma placa maciça de MDF e retira apenas a quantidade necessária para a produção das peças. É inevitável a geração de resíduos em um processo de fabricação subtrativo, mesmo com a utilização de recursos de aninhamento (nesting) das peças para a otimização da quantidade de material disponível. Para minimizar a geração de resíduos no processo de corte a laser foi implantada uma política de reaproveitamento dos resíduos para outros projetos que se encontram em andamento, e uma parte significativa foi reapropriada.

Com relação à estabilidade da estrutura, pode-se afirmar que o principal componente de estabilidade do sistema é a bateria estacionária de $9 \mathrm{v}$. O seu peso elevado associado à sua posição na base do mecanismo cria um ponto de apoio firme que permite que a haste e os módulos fotovoltaicos se movimentem dentro das demandas exigidas sem causar desequilíbrio crítico ao sistema e assim dispensando o uso de uma fixação na mesa ou no solo.

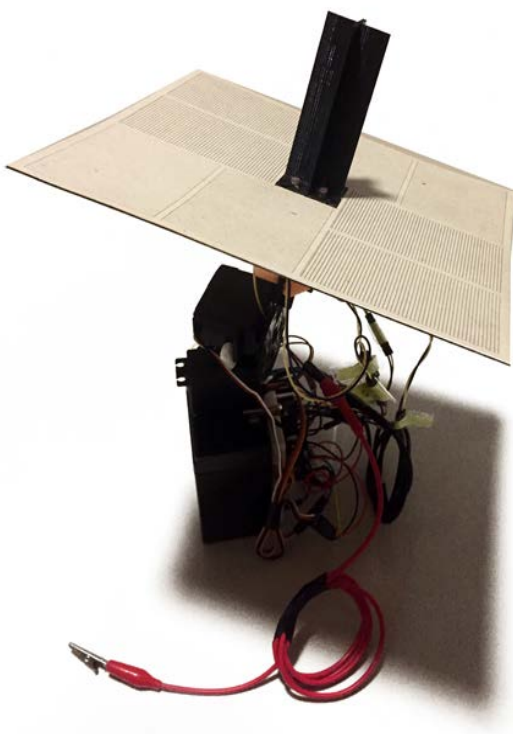

Figura 05: Imagem do Girassol Digital. Fonte: autores

Um dos problemas construtivos frequentes é a desconexão dos circuitos e componentes eletrônicos durante a o funcionamento. Se a movimentação da haste é muito acelerada a vibração do sistema pode acarretar a desconexão de pontos isolados da conexão tanto na protoboard quando nas entradas do Arduino. Trata-se de um problema que também se relaciona ao tipo de fiação utilizada, denominada tecnicamente de jumpers. Os jumpers normalmente utilizados para projetos com Arduino são bastante rígidos, aos moldes de um arame fino. Essa rigidez não é prática em projetos com muitas articulações porque eles não acompanham a movimentação das peças e transmitem essa tensão para os conectores, ocasionando sua soltura. A haste principal do sistema é comandada por dois servo-motores com raio de giro de 180 graus. Neste sentido a haste reproduz essa rotação nos eixos verticais e horizontais, levando com isso toda fiação de ligação dos sensores LDR. Para solucionar esse problema deverão ser substituídos os jumpers por outros modelos mais flexíveis, desde que atendidas as especificações de transmissão elétricas.

Com relação ao tempo de resposta a programação do Girassol Digital obteve uma performance acima da necessidade real. A capacidade de processamento do microcontrolador Arduino Uno permitiu pôr em funcionamento um sistema cujos loops de análise de dados de input e autorregulação (output) fosse obtido a cada 50 milissegundos, o que equivale a 0.05 segundos. Isso faz com que o sistema possa identificar a se movimentar com grande velocidade em direção à uma fonte de luz. No entanto, devido à baixa velocidade relativa que a luz solar se altera em relação a um ponto na superfície da Terra, é possível manter a performance do sistema aumentando intencionalmente o tempo de processamento e resposta sem comprometer a 
performance do mecanismo. Acredita-se assim conseguir uma economia considerável de energia para mantê-lo em funcionamento.

\section{CONCLUSÕES}

O projeto do Girassol digital permitiu revelar a viabilidade de utilização de tecnologias de código aberto para o desenvolvimento de sistemas associados à sustentabilidade ambiental, utilizando uma política de fabricação de baixo custo, baixo desperdício, totalmente engajada como o contexto de ação de arquitetos e designers. Para além das questões relacionadas à viabilidade técnicas, espera-se que com a realização de testes futuros de performance, seja possível comprovar qual o ganho energético oferecido pelo seguidor solar em comparação com os coletores solares estáticos.

A iniciativa em produzir este sistema vai além de um objetivo apenas de performance, mas é parte do interesse direto no desenvolvimento tecnológico brasileiro e na democratização e consequente barateamento no acesso a sistemas de alta tecnologia em todas as camadas populacionais.

\section{AGRADECIMENTOS}

Nossos agradecimentos vão para a Fundação de Amparo à Pesquisa e Inovação do Espírito Santo (FAPES) pelo financiamento de projeto de pesquisa, à Pró-Reitoria de Pesquisa e Pós-Graduação (PRPPG) pelas bolsas de Iniciação Científica, ao Programa de Pós-Graduação em Arquitetura e Urbanismo (Mestrado e Doutorado PPGAU) pelo apoio científico, ao Departamento de Arquitetura e Urbanismo (DAU) pelo apoio na sessão das instalações físicas para o desenvolvimentos dos projetos, e a todos os membros do Grupo de Pesquisas Conexão VIX, inclusos aqueles que já se formaram, titularam e que, direta ou indiretamente, contribuíram com sua presença e participação nas atividades realizadas no laboratório.

\section{REFERÊNCIAS}

Arruda, A. (2018). Métodos e Processos em Biônica e Biomimética: a Revolução Tecnológica pela Natureza. São Paulo: Editora Edgar Blucher.

Atomo Technologies (2020). Seguidor Solar (Brazo Robotico Solar). [online] $\quad$ Retrieved from: http://blog.atomogt.com/2015/09/seguidor-solar-brazorobotico-solar.html. Accessed 06jul2020.

Benyus, J. M. (2003) Biomimética: Inovação inspirada pela natureza. $1^{\text {a }}$ ed. São Paulo: Editora Cultrix. (APA)

Blaszczak, V. (2017). Análise de eficiência de painel fotovoltaico com sistema tracker seguidor solar. \{online\}. Retrieved from: https://rd.uffs.edu.br/bitstream/prefix/1695/1/BLASZCZAK.pdf . Accessed 15may2018.

Bondarik, R., Pilatti, L., Horst, D. (2018). Uma visão geral sobre o potencial de geração de energias renováveis no brasil. Interciencia, 43(10),680-688. Retrieved from: https://www.redalyc.org/articulo.oa?id=339/33957861002. Accessed 25mar2020. ISSN: 0378-1844

Dresch, A., Lacerda, D., Miguel, P. (2015) A Distinctive Analysis of Case Study, Action Research and Design Science Research. Revista brasileira de gestão de negócios, Vol.17(56), pp.1116-1133

Noble, J. (2009). Programming Interactivity. Sebastopol: O'Reilly.

O'Sullivan, D. \& Igoe, T. (2004). Physical Computing: sensing and controlling the physical world with computers, Boston, Thomson.

Oliveira, M. (2008). Análise do desempenho de um gerador fotovoltaico com seguidor solar azimutal. \{online\}. Retrieved from:

http://www.lume.ufrgs.br/bitstream/handle/10183/14737/0006 68141.pdf?sequence=1. Accessed 25may2018.

Pangaro, P.; Dubberly, H. (2011) Introduction to Cybernetics and the Design of Systems. Retrieved from: https://www.pangaro.com/CUSO2014/Cybernetics_Book_of_ Models-v4.6b-complete.pdf. Accessed: 17 out2013

Portoghesi, P. (1993). Natura e Architettura. Itália: Skira.

Ramos, R.; Domingues, Q.; Ricardi, F.; Hübler, P; Bertagnolli, S. (2020) Girassol Autômato: desenvolvendo um dispositivo robótico utilizando a plataforma arduino para otimização do aproveitamento de energia solar. [online] Retrieved from: http://sistemaolimpo.org/midias/uploads/7cfc52da517e0ff0f33 e4da4f2a437db.pdf. Accessed: 24jun2020.

Rebello, Y. (2000). A Concepção Estrutural e a Arquitetura. São Paulo: Zigurate

Rocha, B. (2015) Complexidade e Improvisação em Arquitetura. Tese (Doutorado em Arquitetura e Urbanismo) - Faculdade de Arquitetura e Urbanismo, Universidade de São Paulo, São Paulo.

Wiener, N. (1970) Cibernética: ou controle e comunicação no animal e na máquina. São Paulo: Polígono e Universidade de São Paulo. 